\title{
Le rôle du contexte dans le jugement de pertinence en situation de repérage d'information
}

\section{The Influence of the Context When Evaluating Relevancy during Information Retrieval \\ La función del contexto en la concepción de pertinencia de la situación en la localización de la información}

\section{Martin Boucher}

Volume 59, numéro 1, janvier-mars 2013

URI : https://id.erudit.org/iderudit/1033116ar

DOI : https://doi.org/10.7202/1033116ar

Aller au sommaire du numéro

Éditeur(s)

Association pour l'avancement des sciences et des techniques de la documentation (ASTED)

ISSN

0315-2340 (imprimé)

2291-8949 (numérique)

Découvrir la revue

Citer cet article

Boucher, M. (2013). Le rôle du contexte dans le jugement de pertinence en situation de repérage d'information. Documentation et bibliothèques, 59(1), 5-16. https://doi.org/10.7202/1033116ar
Résumé de l'article

Cet article propose de cerner le concept de contexte en recherche d'information et de mieux comprendre son impact sur la définition de la pertinence et de son mode d'évaluation principal par l'usager, à savoir le jugement de pertinence. Après avoir abordé le concept de contexte, nous explorerons les principales définitions du concept de pertinence ainsi que quelques modèles théoriques qui démontrent l'influence grandissante de l'approche sociotechnique sur ces modèles, soit l'intégration des composantes humaines et techniques ainsi que leurs interactions. Par la suite, nous présenterons certains critères utilisés pour juger de la pertinence des résultats de recherche et nous verrons comment ces critères peuvent être affectés par différentes dimensions du contexte. Enfin, nous soulignerons le caractère dynamique et multidimensionnel des concepts de pertinence et de contexte.
Tous droits réservés (c) Association pour l'avancement des sciences et des techniques de la documentation (ASTED), 2013
Ce document est protégé par la loi sur le droit d'auteur. L'utilisation des services d’Érudit (y compris la reproduction) est assujettie à sa politique d'utilisation que vous pouvez consulter en ligne.

https://apropos.erudit.org/fr/usagers/politique-dutilisation/ 


\title{
Le rôle du contexte dans le jugement de pertinence en situation de repérage d'information
}

\author{
MARTIN BOUCHER \\ Étudiant au doctorat en sciences de l'information \\ EBSI, Université de Montréal \\ martin.boucher@umontreal.ca
}

\section{RÉSUMÉ | ABSTRACTS | RESUME}

Cet article propose de cerner le concept de contexte en recherche d'information et de mieux comprendre son impact sur la définition de la pertinence et de son mode d'évaluation principal par l'usager, à savoir le jugement de pertinence. Après avoir abordé le concept de contexte, nous explorerons les principales définitions $d u$ concept de pertinence ainsi que quelques modèles théoriques qui démontrent l'influence grandissante de l'approche sociotechnique sur ces modèles, soit l'intégration des composantes humaines et techniques ainsi que leurs interactions. Par la suite, nous présenterons certains critères utilisés pour juger de la pertinence des résultats de recherche et nous verrons comment ces critères peuvent être affectés par différentes dimensions du contexte. Enfin, nous soulignerons le caractère dynamique et multidimensionnel des concepts de pertinence et de contexte.

\section{The Influence of the Context When Evaluating Relevancy during Information Retrieval}

The intent of this article is to define the concept of context during information retrieval and to better understand its impact on the definition of relevancy and its evaluation by the user, namely the assessment of relevancy. Following a discussion of the concept of context, the author explores the principal definitions of relevancy as well as several theoretical models that underscore the increasing influence of the socio-technical approach on these models, specifically the integration of human and technical components, including their interactions. The author outlines the criteria used to evaluate the relevancy of the research results and describes how these criteria can be influenced by the different dimensions of the context. Finally, he underscores the dynamic and multidimensional character of the concepts of relevancy and context.

\section{La función del contexto en la concepción de pertinencia de la situación en la localización de la información}

Este artículo tiene como objetivo delimitar el concepto de contexto en la búsqueda de la información y comprender mejor su impacto en la definición de pertinencia y su modo de evaluación principal por parte del usuario, es decir, la concepción del término pertinencia. Tras abordar el concepto de contexto, analizaremos las principales definiciones del concepto de pertinencia, así como algunos modelos teóricos que ejercen una influencia creciente en el enfoque sociotécnico de estos modelos, es decir la integración de los componentes humanos y técnicos y sus interacciones. Luego, presentaremos determinados criterios utilizados para evaluar la pertinencia de los resultados de investigación y observaremos de qué forma estos criterios pueden verse afectados por diferentes aspectos del contexto. Por último, destacaremos el carácter dinámico y multidimensional de los conceptos de pertinencia y de contexto.

\section{Introduction}

L

ES DIFFÉRENTS MODÈLES DU COMPORTEMENT informationnel proposés entre autres par Wilson (1999), Kuhlthau (1991) ou Ingwersen (1996) montrent que la recherche d'information fait parfois intervenir plusieurs stratégies de recherche selon le type de recherche ou le stade de recherche auquel l'usager est rendu. Parmi ces stratégies ou modes de recherche faisant intervenir un système d'information, on retrouve l'exploration, la navigation, le repérage d'information (ou la recherche par mots-clés), le berrypicking et même la découverte fortuite (ou serendipity) (White \& Roth 2009).

Les systèmes de recherche d'information de nature exploratoire, tels que décrits par Marchionini (2006) et White et Roth (2009), sont fondés sur un certain nombre de caractéristiques, comme par exemple le support à la construction et au raffinement de requêtes, le filtrage des résultats par facettes et la visualisation des résultats de recherche pour permettre l'analyse et la prise de décision. Une autre caractéristique fondamentale de ces systèmes est la prise en compte du contexte de l'usager (White \& Roth 2009). La capture du contexte peut alors permettre au système de proposer des documents pertinents pour l'usager. Or, la notion de contexte n'est pas bien définie par White et Roth ; c'est aussi le cas pour la notion de pertinence, souvent associée à une mesure de nature algorithmique basée sur la saillance d'un ou plusieurs termes d'une requête dans les documents d'une collection.

Cet article propose, à partir d'une revue de littérature sélective ${ }^{1}$, de cerner le concept de contexte en recherche d'information et de mieux comprendre son impact sur la définition de la pertinence et de son mode d'évaluation principal par l'usager, à savoir le jugement de pertinence. Malgré son importance dans la recherche en sciences de l'information, l'évaluation des systèmes de repérage (et les moyens mis en œuvre pour

1. Cet article est le fruit d'un travail de synthèse réalisé dans le cadre d'un séminaire doctoral et axé autour d'une douzaine de textes extraits de la littérature scientifique et professionnelle. L'objectif de ce travail était double : présenter quelques concepts, modèles ou théories utilisés par les chercheurs dans le domaine des sciences de l'information sur un sujet donné et extraire des éléments utilisables pour le projet de recherche doctoral. 
l'opérationnaliser) ne sera pas couverte dans la présente analyse. Enfin, cet essai ne prétend pas à l'exhaustivité mais cherche plutôt à présenter différents points de vue sur les questions de contexte, de pertinence et de jugement de pertinence et à voir comment ces concepts s'arriment entre eux.

Nous présentons d'abord deux travaux importants qui traitent de la notion de contexte, ce qui nous permettra d'aborder dans la section suivante le concept de pertinence à travers différentes théories et modèles qui tentent de le définir. Nous terminerons par un survol de la question du jugement de pertinence et de l'impact du contexte sur ce jugement.

\section{Contexte et recherche d'information}

Les années 1980 et 1990 ont vu émerger de nombreuses contributions théoriques et empiriques sur le comportement informationnel, dont celles de Belkin (1993), Kuhlthau (1991), Wilson (1999), Bates (1989) et Ingwersen (1996). Tous ces chercheurs ont suggéré que le processus de recherche d'information (Information Seeking ou IS) est beaucoup plus complexe que celui d'extraction de l'information (communément appelé Information Retrieval ou IR). Le nouveau paradigme de recherche centré sur l'utilisateur a non seulement permis de dégager l'importance des pratiques informationnelles d'un point de vue humain mais aussi de considérer ces pratiques comme un processus prenant place dans des situations ou contextes spécifiques (Courtright 2007).

La notion de contexte, bien qu'abordée par plusieurs auteurs, ne nous apparaît cependant pas définie de manière convaincante, laissant la place à de nombreuses interprétations. Par exemple, pour Mizzaro, le contexte est "tout ce qui ne concerne pas le sujet et la tâche mais cependant affecte la manière dont la recherche se déroule et l'évaluation des résultats " (1997, 811 , notre trad.). Pour plusieurs chercheurs, le contexte se définit comme un "cadre de référence" pour les pratiques informationnelles (Courtright, 2007). Qui plus est, Courtright différencie le contexte de la notion de "situation" qui concerne davantage l'environnement immédiat et dynamique de l'usager. Pour elle, le contexte est plus large et « englobe » la situation. Malgré cette distinction, Courtright note que le concept de contexte est plutôt informe et insaisissable. Néanmoins, elle propose dans sa revue de littérature d'en définir les contours et les éléments qui le constituent et expose une catégorisation des différentes théories et modèles du concept de contexte (2007). Nous présenterons cette délimitation du contexte et la catégorisation de Courtright, que nous rapprocherons par la suite du modèle stratifié de Ingwersen et Järvelin (2005).

\section{Délimitation du concept de contexte}

Pour définir le contour du concept de contexte, Courtright distingue, à partir d'une recension de la littérature, deux catégories de pratiques informationnelles : la première est liée aux activités des organisations et la seconde est issue de la vie de tous les jours (2007). Dans le premier cas, les pratiques sont nettement plus encadrées en raison d'une plus grande stabilité de ce que l'auteure appelle les « champs d'information ». Plusieurs modèles tentent d'ailleurs de décrire le contexte organisationnel, tels que le «information use environment » ou IUE de Taylor (Courtright 2007, 278), ou le modèle des écologies de l'information de Nardi et O'Day (Courtright 2007, 278). Ces modèles s'attardent aux flux et aux usages de l'information par des professionnels dans leur environnement de travail. Courtright mentionne également des études centrées davantage sur les rôles en situation de travail.

En dehors des organisations, les contextes sont beaucoup plus difficiles à circonscrire, notamment lorsqu'il est question d'étudier les réseaux d'information. Courtright présente quelques modèles conceptuels issus de théories sociologiques, dont le «information ground » qui représente les environnements temporaires de personnes qui se rejoignent pour une raison singulière et qui favorisent l'échange spontané et fortuit d'informations. Parmi ces contextes, on peut retrouver les salons de coiffure, les cours donnés en bibliothèques et les environnements de travail (Courtright 2007).

Courtright mentionne deux autres modèles de pratiques informationnelles de la vie quotidienne par lesquels les chercheurs tentent de délimiter le contexte : le "small-world theory" de Chatman (2000) et le modèle de Savolainen (1995) basé sur l'« habitus» de Bourdieu. Le premier est caractérisé par l'aspect profondément social des pratiques informationnelles alors que le second décrit le contexte en fonction du mode de vie qui façonne les choix et les décisions (Courtright 2007). Enfin, Courtright présente le modèle conceptuel de Lievrouw (2001) qui prend en compte l'évolution dynamique des pratiques informationnelles des acteurs et qui postule l'existence d'une multiplicité d'environnements informationnels qui peuvent se superposer selon des impératifs et des activités de recherche.

\section{Facteurs contextuels}

À travers sa revue de littérature, Courtright identifie une liste de facteurs contextuels qui façonnent les pratiques informationnelles et qui peuvent avoir un impact significatif sur le jugement de pertinence, sujet que nous aborderons en détail plus loin.

Les recherches empiriques et théoriques font état de relations entre des éléments contextuels et des activités informationnelles, à la fois dans le cadre de pratiques organisationnelles et de pratiques quotidiennes. 
La liste suivante résume ces différents éléments ou facteurs tels que catégorisés par Courtright :

- Règles et types de ressources ;

- Culture (organisationnelle ou sociale);

- Réseau social ou capital social ;

- Normes sociales ou autorité sociale ;

- Exigence collaborative en environnement de travail;

- Tâche ou situation ;

- Rôle ou domaine de travail ;

- Activités humaines.

Tous ces facteurs sont à même d'influencer la perception et l'évaluation de la pertinence lors de l'activité de recherche d'information. Parmi ces facteurs, la tâche est considérée comme étant la principale source d'influence dans nombre d'études sur les pratiques organisationnelles, alors que la "situation" est davantage associée à des recherches portant sur les pratiques hors du milieu de travail. En outre, plusieurs études attribuent une grande part de la variabilité dans les pratiques informationnelles au rôle ou domaine de travail (Courtright 2007).

Enfin, Courtright insiste sur le rôle double des technologies de l'information (TI) comme facteur contextuel. D'une part, les TI constituent un contexte à part entière dans les pratiques informationnelles, allant jusqu'à modifier ces pratiques. D'autre part, nombre d'éléments contextuels externes aux TI ont le potentiel d'influencer l'adoption de ces technologies.

\section{Statut ontologique du contexte}

La compréhension du statut du contexte en relation avec le chercheur a fait l'objet de modèles qui ont été classés par Courtright en cinq catégories : le contexte comme contenant, la personne en contexte, l'acteur social, l'imbrication et enfin le changement (2007).

Le contexte, s'il est défini selon une réalité objective, peut être décrit en fonction d'un ensemble d'entités stables et délimitées, conceptualisées indépendamment des activités du participant. Dans cette vision positiviste, le contexte est un contenant ou une toile de fond pour les pratiques informationnelles qui nivelle les différences individuelles et ne tient pas compte de la variabilité des pratiques.

Par opposition au contexte "comme contenant", la «personne en contexte» est une approche centrée sur les activités informationnelles en relation avec les variables et influences perçues et construites par l'usager (Courtright 2007). Parmi les modèles couramment cités, notons le modèle imbriqué de Wilson (1999) qui présente le contexte comme une série de couches concentriques, partant des aspects physiologiques, affectifs et émotifs et s'élargissant aux rôles et à l'environnement dans lesquels les besoins informationnels et les actions de recherche s'inscrivent. Parmi les

\section{Figure 1}

Un des modèles de Ingwersen et Järvelin mentionné par Courtright

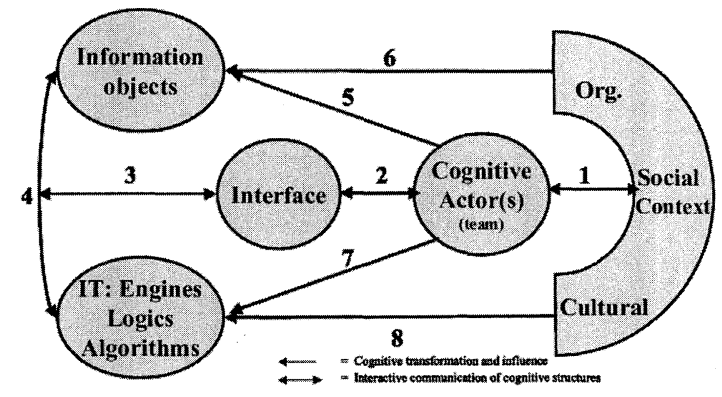

modèles aussi mentionnés par Courtright, notons celui de Ingwersen et Järvelin (Figure 1) qui situe l'acteur au centre du processus de recherche, l'acteur interagissant avec un contexte socioculturel d'une part, et, d'autre part, avec des objets et systèmes d'informations (Ingwersen et Järvelin 2005).

L'acteur évoluant généralement dans un cadre social et construisant l'information au moyen d'interactions sociales notamment, plusieurs critiques ont été soulevées concernant l'exclusion du contexte social de la vision de la personne en contexte. La vision « socioépistémologique " considère plutôt l'acteur en regard de son appartenance à une communauté sociale partageant des connaissances, des caractéristiques ou des attentes qui sont internalisées. Courtright (2007) mentionne que l'imbrication de l'acteur social dans des contextes encore plus larges (institutions, facteurs technologiques) ne peut se faire au détriment d'une séparation des différents participants. En fait, les contextes sont inter-reliés et interagissent les uns avec les autres. Enfin, cette vision relationnelle du contexte implique le concept de changement, induisant une forme d'«écologie de l'information ». Dans un tel système ou écologie, les facteurs ou composantes du contexte sont en constante évolution (2007).

\section{Dimensions du contexte}

Une représentation d'une telle écologie de l'information est illustrée par Ingwersen et Järvelin (2005) dans leur modèle imbriqué de stratification du contexte (Figure 2). Ce modèle, conçu pour décrire un cadre de recherche pour les systèmes de recherche et de repérage d'information en contexte, présente les différentes strates de contexte telles que perçues par les différents acteurs impliqués dans le processus de recherche d'information.

Pour Ingwersen et Järvelin, les documents, les composantes informatiques et les interfaces sont autant d'objets qui composent l'espace documentaire de recherche. Ces éléments peuvent être décomposés en lignes de code ou en structures textuelles 
Figure 2

Modèle imbriqué de stratification du contexte (selon Ingwersen \& Järvelin 2005, 281)

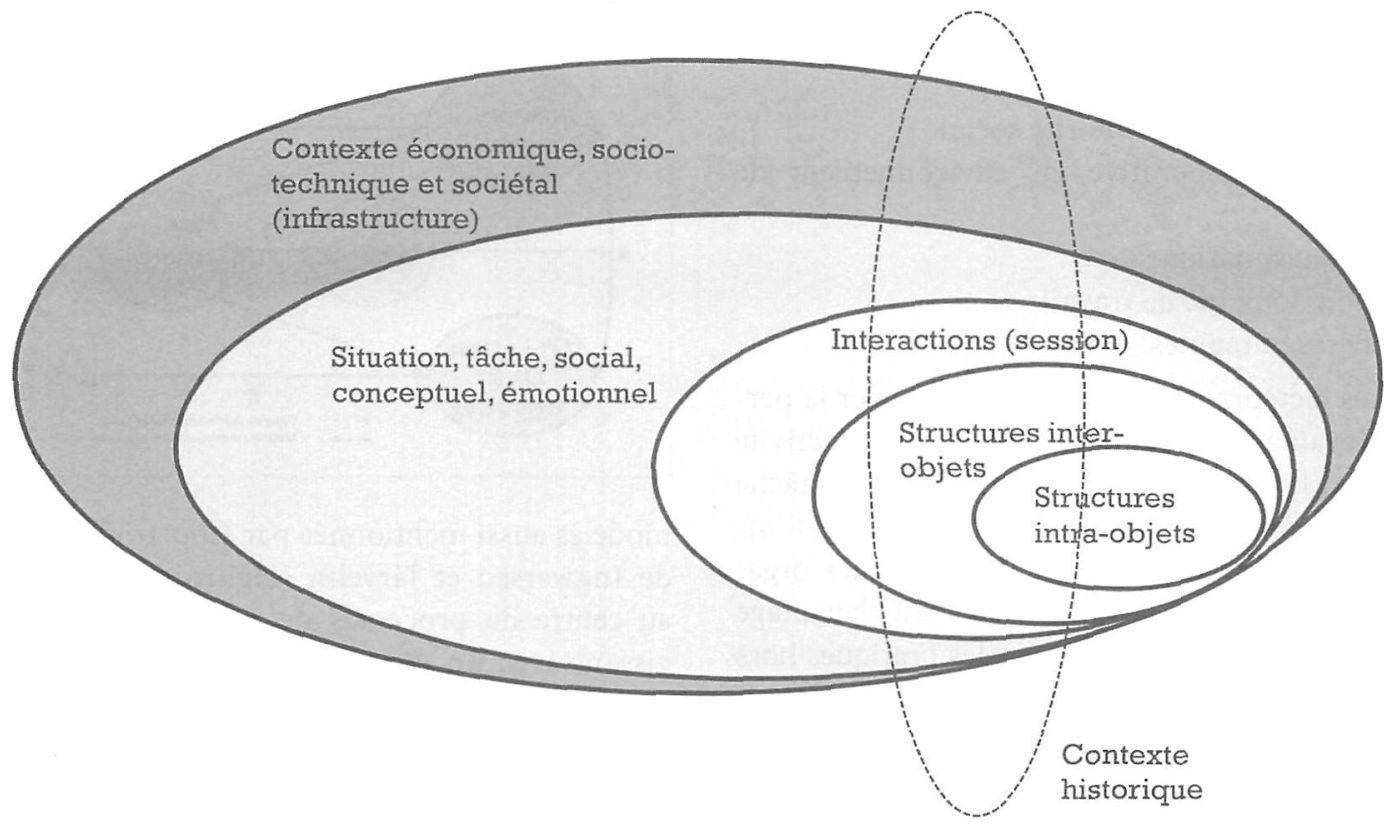

(mots, paragraphes, etc.) par exemple, formant ainsi le contexte « intra-objets ».

Les structures "inter-objets » concernent les relations entre les différents objets. Les liens entrants ou sortants et le référencement croisé (un document cite ou est cité par un ou plusieurs autres documents) sont autant d'exemples de ce type de structure.

La strate médiane représente le contexte des interactions entre l'usager et le système, façonné à la fois par les composantes ou objets placés au cœur du modèle (structures inter-objets et intra-objets) et par les composantes cognitives et émotives des strates supérieures. En effet, les composantes informatiques, les interfaces et les documents orientent le type d'interaction et de suivi dans le temps, au-delà de la simple collecte de données sur l'usager :

"Interagir avec les chercheurs signifie, pour le systeme, davantage que simplement capturer les données de requête. Plutôt, l'interaction, dans le temps, du chercheur avec un système forme un réseau riche d'information potentielle en regard des préférences, du style, de l'expérience et de la connaissance aussi bien que de l'intérêt."

(Ingwersen et Järvelin 2005, 280, notre trad.).

Les deux dernières strates renvoient à des composantes plus larges du système et de l'environnement. Dans le premier cas, la strate « situation, système, tâche, etc. » intègre la plupart des composantes du comportement informationnel, à la fois du point de vue des expériences cognitives et émotives de l'usager et du point des influences extérieures immédiates.
La dernière strate modélise les influences économiques et sociotechniques des différents acteurs. Elle concerne ainsi une dimension relevant davantage de l'infrastructure sociétale dans laquelle prennent place les autres strates (Ingwersen et Järvelin 2005). En effet, un système de repérage spécifique (et les documents auxquels il donne accès) se situe dans un environnement et une infrastructure définis par une culture, un contexte économique ou des usages et pratiques informationnels précis. L'usager est aussi teinté par ces mêmes influences, qu'il en soit conscient ou non.

Enfin, toutes ces strates sont soumises au contexte historique des expériences de tous les acteurs participant au modèle. Ces expériences, inscrites dans le temps, engendrent et façonnent les attentes des acteurs. En outre, le temps, comme forme particulière de contexte, influence toutes les activités et processus de recherche et de repérage de l'information.

En conclusion, Ingwersen et Järvelin sous-tendent que la recherche et le repérage d'information en contexte ne peuvent être considérés uniquement sous l'angle des "contextes des chercheurs" ou du «chercheur comme contexte", mais concernent aussi l'interaction entre les documents et la plateforme (système) en regard de domaines d'applications et de tâches spécifiques (2005). Le système lui-même doit donc être compris et analysé comme composante du contexte, au même titre que les autres composantes que sont entre autres l'usager, ses connaissances, l'environnement, la situation ou la tâche.

Le modèle stratifié de Ingwersen et Järvelin et la catégorisation des modèles de contexte de Courtright mettent clairement en évidence le caractère 
multidimensionnel du concept de contexte ainsi que sa nature dynamique. Les différents types de contexte évoqués, qu'ils proviennent du système, de l'usager ou de l'environnement, se superposent et s'influencent mutuellement, expliquant ainsi la variabilité du comportement informationnel et démontrant la complexité de la tâche de développement des systèmes de repérage de l'information en contexte.

\section{Pertinence : théories et modèles}

Le concept de pertinence, tel que défini par rapport aux systèmes de repérage de l'information, est intimement lié au développement des sciences de l'information, et ce depuis ses tout débuts, comme le fait remarquer Saracevic (1975). Mizzaro en dresse d'ailleurs un historique en 1997, situant alors ses trois différentes périodes, soit celle d'avant 1958 marquée par les premiers débats sur le concept, celle de 1959 à 1976 et celle qui débute en 1977, marquée progressivement par une prédominance des recherches sur le comportement informationnel.

Durant ces trois périodes, plusieurs définitions de la pertinence ont été proposées, la plupart caractérisées par une dimension relationnelle. Selon Saracevic, la pertinence est une mesure d'efficacité du contact entre la source et la destination dans le processus de communication : «La pertinence est, ainsi, un aspect fondamental de la communication humaine. " $(1975,324$, notre trad.). $\mathrm{Xu}$ et Chen (2006) notent que, selon Saracevic, la pertinence en général est conceptualisée comme le jugement de l'usager sur la relation entre un document et son besoin informationnel. Pour Harter (1992), la pertinence (relevance) est une relation entre un document et une question ou un sujet.

La notion de pertinence a cependant évolué entre ses premières définitions centrées davantage sur un paradigme positiviste (centré système) vers un paradigme centré usager, incluant progressivement des éléments empruntant aux études et théories sur le comportement informationnel. Borlund écrit, à propos du changement de paradigme dans la définition de pertinence: "Le résultat de la révolution de la pertinence est une acceptation croissante que la pertinence devrait être jugée en relation avec le besoin informationnel plutôt qu'avec la requête." (2003, 923, notre trad.).

Nous présentons, dans les lignes qui suivent, quelques définitions du concept de pertinence, notamment à travers ses différentes manifestations, ainsi que quelques modèles théoriques.

\section{Les manifestations de la pertinence}

La pertinence se manifeste ou peut être comprise de plusieurs manières et suivant plusieurs paradigmes. Le caractère objectif-subjectif de la pertinence a été repris par maints chercheurs, notamment Borlund
(2003), Cosijn et Ingwersen (200o) et Saracevic (1996). Nous présenterons donc ici le concept de pertinence selon les différentes définitions qui lui ont été attribuées ainsi que selon son caractère objectif ou subjectif.

\section{La pertinence objective}

Le caractère objectif de la pertinence se distingue par une conception ancrée dans une tradition nettement positiviste, que ce soit par une définition algorithmique (ou calculatoire) ou par adéquation objective entre une requête et le contenu d'un document.

\section{Pertinence orientée système}

La pertinence orientée système (ou algorithmique), qui tire ses origines des premiers systèmes de repérage d'information basés sur la concordance entre une requête et les termes indexés d'un document, est la plus facile à décrire et, selon Saracevic (1996), correspond davantage à une propriété du système de repérage. Ce paradigme, défini par le modèle Cranfield à la fin des années 1950 et au début des années 1960, propose de n'évaluer que la portion concernant la manière dont le système acquiert, représente, organise et compare les textes, sans tenir compte des interactions de l'usager.

En 1996, Saracevic note que cette approche est encore présente sinon omniprésente dans les systèmes de repérage d'information des années 1990 ; il en donne pour preuve l'initiative majeure d'évaluation des systèmes de recherche d'information, le Text REtrieval Conference (TREC) ${ }^{2}$ qui a vu le jour en 1992 et qui se poursuit encore aujourd'hui. Cette compétition, du moins durant ses premières années, utilise un protocole largement inspiré du modèle Cranfield où les résultats sont évalués selon les mesures standards de précision et de rappel.

De manière générale, la pertinence algorithmique est considérée comme un concept statique et objectif (Borlund 2003).

\section{Topicalité}

Harter (1992) considère la topicalité (topicality) comme une définition objective de la pertinence, où un document est dit pertinent s'il traite du sujet de la requête. Pour lui, cette adéquation entre le sujet du document et une question ou une requête peut faire l'objet d'une évaluation objective par des experts du domaine. Or, selon Borlund (2003), l'évaluation, pour être objective, ne peut être faite que sur le contenu manifeste du document, à savoir entre les mots qu'il contient et les mots de la requête.

\footnotetext{
2. <http://trec.nist.gov>.
} 


\section{La pertinence subjective}

\section{La pertinence thématique ou « sujet »}

La littérature (anglophone surtout) réfère à ce type de pertinence sous l'appellation "topical relevance " ou "aboutness", qui ne doit pas être confondue avec «topicality». À défaut d'un vocable plus approprié, nous emploierons, pour la suite, la notion de pertinence thématique bien que ce type de pertinence se réfère davantage au sujet de la requête ou du document.

Saracevic (1996) décrit la pertinence thématique comme la relation entre un sujet exprimé dans une requête et le sujet couvert par des textes récupérés. Pour Borlund (2003), il n'est plus question d'établir uniquement une correspondance entre les mots de la requête et ceux des documents, mais bien de recourir à l'évaluation de cette relation par l'usager Elle nomme d'ailleurs ce type de pertinence "intellectual topicality» de manière à la distinguer de la topicalité qui est généralement considérée comme un type de pertinence objective.

Cosijn et Ingwersen (2000) mentionnent le rôle de l'intentionnalité dans l'évaluation de la pertinence thématique. En situation d'évaluation du sujet couvert par un document, un usager ou un évaluateur d'un système de repérage auront nécessairement des intentions différentes, le premier étant confronté à un besoin informationnel spécifique et le second mis en situation d'évaluation de manière plus beaucoup plus objective, sans intentionnalité.

\section{Pertinence cognitive}

La notion de pertinence cognitive réfère à la relation entre les documents et l'état de connaissance et le besoin informationnel cognitif de l'usager (Saracevic, 1996). Certains auteurs, comme Saracevic (1975, 1996), Borlund (2003) ou Cosijn et Ingwersen (2000) parlent aussi de « justesse » (en anglais pertinence).

En 1975, Saracevic constate qu'il n'existe aucune étude sur le sujet de la pertinence cognitive, qu'il nomme "destination's knowledge view of relevance", référant ainsi à la relation entre la connaissance détenue par un individu et la connaissance (ou littérature) disponible sur le sujet (subject knowledge). Vingt ans plus tard, en 1996, il mentionne quelques critères qui, selon lui, permettent d'inférer ce type de pertinence: correspondance cognitive, "informativité », nouveauté, qualité de l'information, etc.

\section{Pertinence psychologique}

Stephen Harter (1992) reprend, dans le contexte des sciences de l'information, le concept de pertinence psychologique proposé par Sperber et Wilson dans leur livre Relevance: Communication and cognition de 1986.
Ces derniers, qui s'intéressent uniquement à la communication verbale, définissent le contexte comme un construit psychologique qui représente les hypothèses de l'auditeur à propos du monde, à un moment donné. L'idée de contexte est dynamique et soumise à des "effets contextuels» produits par des processus déductifs d'effacement (une hypothèse peut être effacée du contexte à tout moment) ou de renforcement (positif ou négatif). Pour Sperber et Wilson, une hypothèse est pertinente lorsque celle-ci engendre un effet contextuel. De la même manière, un phénomène (ou stimuli) est pertinent s'il provoque un changement cognitif.

Harter tente d'appliquer le concept de pertinence psychologique au contexte du repérage d'information (information retrieval). Ainsi, des documents seront considérés psychologiquement pertinents s'ils produisent une réponse cognitive suffisamment forte. Le besoin informationnel, généralement exprimé à l'aide d'une requête faite à un système de repérage, peut alors être considéré comme un contexte initial, c'està-dire l'état cognitif d'un individu qui consulte un système de repérage, à un moment donné (1992). Pour Harter, le concept de pertinence psychologique illustre bien le caractère dynamique du besoin informationnel qui évolue après chaque nouveau document consulté, comme le suggère aussi l'étape d'exploration du processus de recherche de Kuhlthau (1991).

Enfin, faisant appel à la définition d'information comme processus de Buckland (1991), Harter (1992) relie les concepts d'information et de pertinence, statuant qu'une condition nécessaire et suffisante pour la pertinence est le caractère informatif.

\section{Pertinence situationnelle}

Pour Borlund (2003), le jugement de pertinence varie selon la situation ou la tâche. L'auteure mentionne l'article de Patrick Wilson datant de 1973, «Situational relevance ", où ce dernier définit la pertinence situationnelle comme la relation entre l'objet d'information et la vue du monde et de la situation du récepteur de la dite information. Borlund rappelle aussi que, pour Patrick Wilson, l'information comme processus est l'élément dynamique du processus de jugement de pertinence. La pertinence situationnelle est centrée sur l'usager, dynamique et essentiellement empirique. C'est l'expression d'une relation entre la perception d'utilité d'objets d'information récupérés et une situation spécifique de travail.

Reprenant la définition du concept de contexte donnée par Harter (1992), Borlund insiste sur le fait que la pertinence situationnelle partage les deux mêmes conditions nécessaires au dynamisme du besoin informationnel :

1. Une situation donnée qui permet une perception cognitive de cette même situation et qui peut engendrer un besoin informationnel ; 
2. Une information fournie de manière externe. Ainsi, un changement de structure de la connaissance de l'usager peut induire une modification de la perception de son besoin informationnel. L'utilisation de la pertinence situationnelle permet donc de considérer le jugement de pertinence comme un processus cognitif.

Enfin, Borlund qualifie ce type de pertinence comme "usefulness" $(2003$, 915) et note qu'en pratique il est très difficile de le différencier de la pertinence cognitive.

\section{Pertinence motivationnelle}

Proposé par Saracevic en 1996, ce type de pertinence est celui qui fait le moins consensus. Il se définit comme la relation entre les intentions, buts et motivations de l'usager et les textes évalués. Saracevic y inclut aussi une dimension affective. Parmi les critères inférant une pertinence motivationnelle, celui-ci note la satisfaction, le succès et l'accomplissement.

Pour Cosijn et Ingwersen (2000), la pertinence motivationnelle de Saracevic est la plus résolument humaine et sociale. Pour cette raison, ils proposent plutôt de la nommer pertinence «socio-cognitive». En ce qui concerne la dimension affective de ce type de pertinence, Cosijn et Ingwersen considèrent qu'elle recouvre tous les types de pertinence subjective et n'est pas une caractéristique propre à un seul type. Borlund (2003) va plus loin en ne considérant pas ce type de pertinence comme indépendant des autres. En fait, elle ne retient que trois types de pertinence subjective : la pertinence thématique, la pertinence cognitive et la pertinence situationnelle.

\section{Quelques modèles théoriques}

Plusieurs modèles théoriques ont été élaborés afin de relier et expliquer les différents types de pertinence. Nous en explorons ici quelques-uns.

\section{Saracevic (1975)}

Suivant la définition selon laquelle la pertinence constitue une mesure d'efficacité du contact entre la source et la destination dans le processus de communication de la connaissance, Saracevic (1975) identifie plusieurs facteurs et relations impliqués dans cette définition. Ces facteurs sont :

1. La connaissance du sujet (subject knowledge);

2. La littérature sur le sujet (subject literature);

3. Toute autre représentation symbolique ou linguistique ;

4. La source (les fichiers ou documents) etc.;

5. La destination (les fichiers ou documents);

6. Les systèmes d'information;

7. Les environnements, réalités et fonctions ;

8. Les valeurs.
Saracevic mentionne que plusieurs de ces facteurs sont généralement considérés comme étant les seuls facteurs constitutifs de la notion de pertinence, sans tenir compte de l'existence possible d'autres facteurs. Or, pour lui, un cadre de référence pour le concept de pertinence se doit d'inclure au minimum ces facteurs, mais aussi en considérer d'autres.

À partir de ces facteurs, Saracevic élabore un ensemble de vues (views) de la pertinence qui, bien que distinctes, ne sont pas nécessairement indépendantes. Les différentes vues sont :

1. La vue de la connaissance du sujet, qui relie la connaissance sur un sujet à une question (topic) sur le sujet;

2. La vue de la littérature sur le sujet, qui couvre les relations entre le sujet et ses différentes représentations dans la littérature ;

3. La vue logique qui concerne les inférences entre les prémisses sur des questions (topic) et les conclusions d'un sujet ou de la littérature sur celui-ci ;

4. La vue du système qui considère les contenus et processus produits par le système d'information en relation avec le sujet traité, la question et l'usager;

5. La vue du destinataire qui renvoie aux jugements humains ;

6. La vue de la "pertinence» ou connaissance $d u$ destinataire qui établit une relation entre la connaissance disponible du destinataire et la connaissance sur le sujet ;

7. La vue pragmatique qui considère la relation entre le problème immédiat de l'usager et l'information fournie.

\section{Saracevic (1996)}

Vingt ans après la publication de son premier modèle, Saracevic en propose un nouveau en 1996 ; il y réduit à cinq le nombre de manifestations de la pertinence (1996) :

1. Pertinence système ou algorithmique ;

2. Pertinence du sujet traité ou topicalité ;

3. Pertinence cognitive (ou pertinence);

4. Pertinence situationnelle ou utilité ;

5. Pertinence motivationnelle ou affective.

Dans son nouveau modèle, Saracevic introduit un modèle d'interaction stratifié, à la lumière de modèles concurrents comme le modèle d'épisode de Belkin (cité par Saracevic 1996) ou le modèle cognitif d'interaction de Ingwersen (1996) qui tiennent compte des aspects dynamiques de l'interaction entre l'usager et le système (Figure 3). Pour Saracevic, ce modèle permet de définir un système de "pertinences", où la notion de pertinence renvoie à une pluralité de manifestations interdépendantes et dynamiques, modulées par un 


\section{Figure 3}

Modèle stratifié d'interaction dans le processus de repérage d'information (Saracevic 1996)

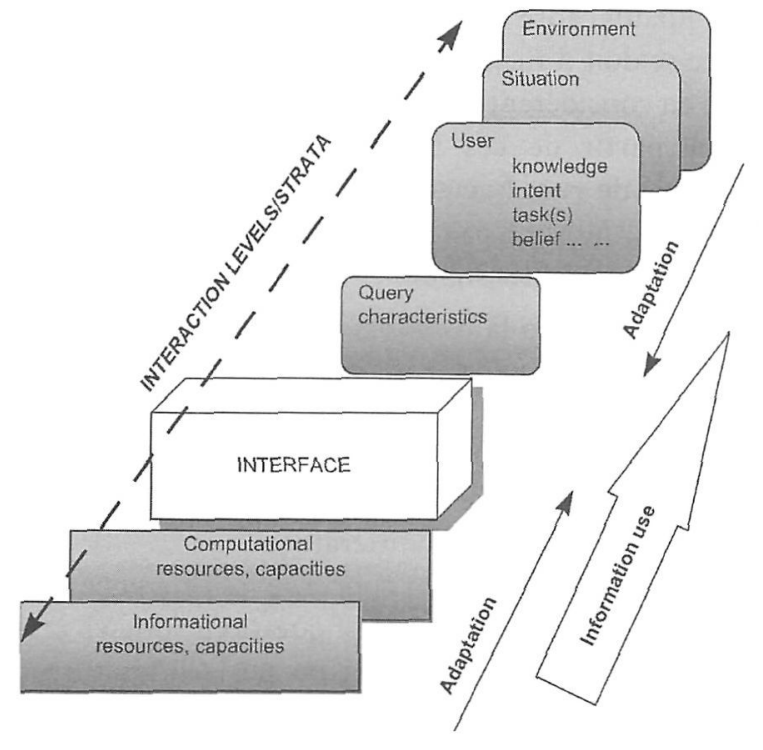

ensemble d'interactions entre les différentes strates du modèle.

Enfin, Saracevic décrit cinq attributs de la pertinence, soit la relation, l'intention, le contexte, l'inférence et l'interaction. Nous y reviendrons.

\section{Mizzaro (1997, 1998)}

Mizzaro (1997) postule qu'il existe différents types de pertinence et non un seul. La pertinence est pour lui l'établissement d'une relation entre deux éléments de deux groupes, soit le groupe des ressources d'information (information, document, substitut du document ou surrogate) et le groupe du besoin informationnel (problème, besoin informationnel, expression verbale du besoin ou request, requête booléenne ou query). Pour Mizzaro, il peut exister une relation entre chaque élément de ces deux groupes, à des moments distincts dans le temps et en relation directe avec les entités sujet (topic), tâche et contexte. Toutes ces relations induisent un ordre partiel (Figure 4) que Mizzaro modélise formellement en 1998.

Dans ce modèle, chaque pertinence peut être vue comme un point dans un espace à quatre dimensions :

1. Les sources d'information, à gauche;

2. Les représentations du problème de l'usager, à droite ;

3. Les composantes sujet, tâche et contexte;

4. Le temps, qui n'apparait pas dans le modèle.

Pour Mizzaro (1998), les deux premières dimensions peuvent être ordonnées mathématiquement :

Surrogate $<$ Document $<$ Information

Query $<$ Request $<$ Information Need $<$ Problem

\section{Figure 4}

L'ordre partiel des pertinences de Mizzaro

(1997, p 812)

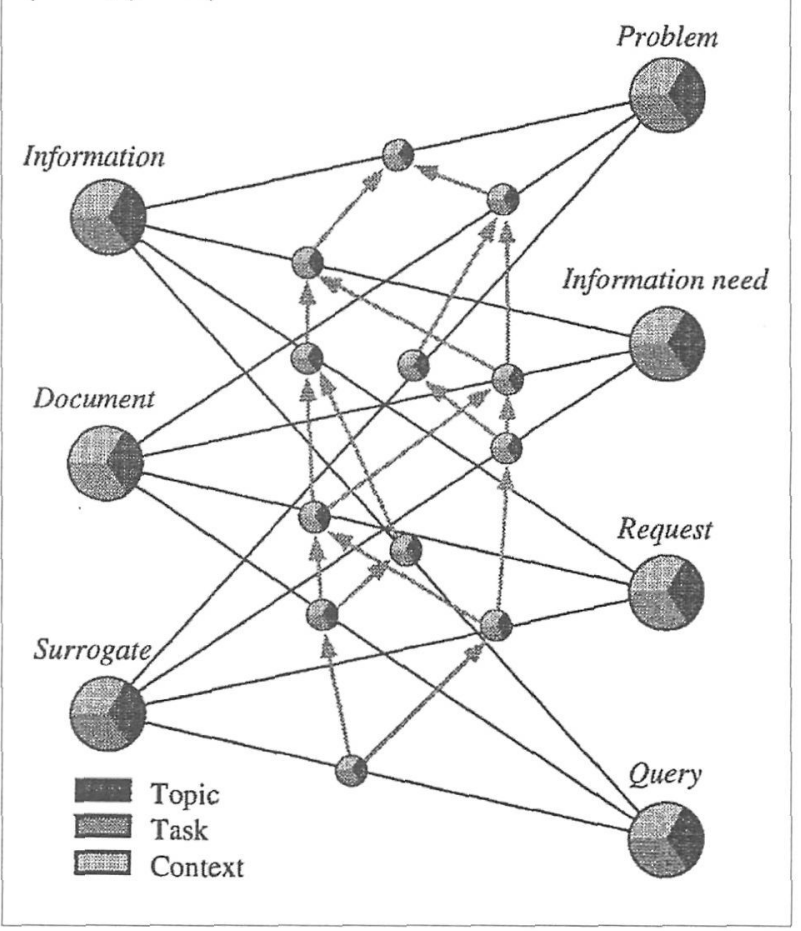

La troisième dimension n'est que partiellement ordonnée.

Ce modèle présente une hiérarchie des éléments constituant les types de pertinence et intègre à la fois des éléments de contexte et la notion de temporalité.

\section{Cosijn et Ingwersen (2000)}

Partant du modèle de Saracevic (1996), Cosijn et Ingwersen (2000) ont modélisé les différentes manifestations de la pertinence par rapport aux attributs de pertinence définis par Saracevic. Ces attributs sont:

- La relation : la pertinence implique une relation, souvent une communication ou un échange ;

- L'intention: la relation dans l'expression de la pertinence implique des intentions telles que des objectifs, des buts, des rôles ou des attentes;

- Le contexte : les intentions proviennent toujours d'un contexte;

- L'inférence: l'évaluation de l'efficacité d'une relation donnée ;

- L'interaction : l'inférence est accomplie dans un processus dynamique d'interaction et les interprétations des autres attributs sont modifiées en fonction des changements cognitifs.

Cosijn et Ingwersen relient les différentes manifestations de la pertinence à ces cinq attributs. Le modèle leur permet de dégager deux conclusions :

1. La pertinence motivationnelle ne peut être vue selon une échelle objective-subjective de la 


\section{Tableau 1}

Résumé des modèles théoriques

\begin{tabular}{|c|c|c|}
\hline Modèle & MANIFESTATIONS & INTERACTION ET DYNAMISME \\
\hline Saracevic (1975) & $\begin{array}{l}\text { Connaissance du sujet } \\
\text { Littérature sur le sujet } \\
\text { Vue logique } \\
\text { Vue du système } \\
\text { Vue du destinataire } \\
\text { Connaissance du destinataire } \\
\text { Vue pragmatique }\end{array}$ & Interdépendance entre les vues de la pertinence. \\
\hline Saracevic (1996) & $\begin{array}{l}\text { Pertinence algorithmique } \\
\text { Pertinence thématique } \\
\text { Pertinence cognitive } \\
\text { Pertinence situationnelle } \\
\text { Pertinence motivationnelle ou affective }\end{array}$ & $\begin{array}{l}\text { Modèle stratifié d'interactions entre } \\
\text { - Environnement } \\
\text { - Situation } \\
\text { - Usager } \\
\text { - Requête } \\
\text { - Ressources technologiques } \\
\text { - Ressources informationnelles }\end{array}$ \\
\hline Mizzaro $(1997,1998)$ & $\begin{array}{l}\text { Toutes les combinaisons de relations entre } \\
\text { - Sources d'information } \\
\text { - Représentation du problème } \\
\text { - Sujet / tâche / contexte } \\
\text { - Temps }\end{array}$ & $\begin{array}{l}\text { Le caractère dynamique de la pertinence est } \\
\text { intrinsèque au modèle de Mizzaro. }\end{array}$ \\
\hline $\begin{array}{l}\text { Cosijn et Ingwersen } \\
(2000)\end{array}$ & $\begin{array}{l}\text { Pertinence algorithmique } \\
\text { Pertinence thématique } \\
\text { Pertinence cognitive } \\
\text { Pertinence situationnelle }\end{array}$ & $\begin{array}{l}\text { L'interaction est un attribut de la pertinence et se } \\
\text { manifeste à travers tous les types de pertinence. } \\
\text { L'interaction s'inscrit dans le temps. }\end{array}$ \\
\hline Borlund (2003) & $\begin{array}{l}\text { Pertinence situationnelle } \\
\text { Pertinence algorithmique } \\
\text { Pertinence thématique } \\
\text { Pertinence cognitive }\end{array}$ & $\begin{array}{l}\text { La pertinence est un concept dynamique et } \\
\text { multidimensionnel. }\end{array}$ \\
\hline
\end{tabular}

pertinence mais plutôt comme un attribut de la pertinence ;

2. La manifestation émotionnelle de la pertinence ne peut être considérée comme une catégorie distincte de la pertinence mais plutôt comme un facteur influençant tous les autres types de pertinence.

Enfin, Cosijn et Ingwersen mentionnent le temps comme facteur déterminant pour les manifestations thématiques et cognitives de la pertinence (2000).

\section{Borlund (2003)}

Parmi les modèles présentés ici, celui de Borlund (2003) est certainement celui qui intègre le plus grand nombre d'éléments influençant la pertinence et le jugement de pertinence. Citant Schamber et al. (1990), Borlund situe son modèle par rapport à trois conclusions importantes :

- La pertinence est un concept cognitif multidimensionnel qui dépend largement de la perception de l'usager sur l'information et son propre besoin informationnel ;

- La pertinence est un concept dynamique qui dépend des jugements des usagers sur la qualité de la relation entre information et besoin informationnel à un moment donné dans le temps ;

- La pertinence est un concept complexe mais mesurable et systématique s'il est conceptualisé et opérationnalisé adéquatement à partir de la perspective de l'usager.

Borlund définit la pertinence comme la relation intellectuelle entre le besoin informationnel intrinsèquement humain et les objets d'information tels qu'ils sont interprétés ou perçus par l'état cognitif d'un usager ou d'un évaluateur (assessor) (Borlund 2003, 915). Son modèle tient compte de la diversité des manifestations de la pertinence et des relations qui les unissent. On constate qu'elle ne traite pas spécifiquement du contexte bien qu'elle fasse référence au Real world et à la tâche de travail.

\section{Synthèse des différents modèles}

À la lumière de ces modèles, une tendance se dessine : celle d'une définition de la pertinence centrée davantage sur des modèles interactifs entre l'usager et le système et sur une composante temporelle omniprésente. La pertinence ne semble plus être définie de manière statique, mais plutôt selon l'écosystème de l'information, dans une perspective nettement sociotechnique. À preuve, la constance dans la prise en compte 
par les différents modèles (surtout les plus récents) du caractère complexe, multidimensionnel et dynamique des différentes manifestations de la pertinence (voir Tableau 1).

\section{Jugement de pertinence}

Contrairement au concept abstrait de "pertinence " qui se traduit sous forme de modèles et de théories, le jugement de pertinence renvoie plutôt à l'évaluation, généralement qualitative, que se fait un usager de la pertinence effective d'un document lors d'un processus de recherche d'information.

Selon Harter (1992), le jugement de pertinence se manifeste lorsqu'un usager, durant une interaction avec un système de repérage d'information, est confronté à un ensemble de résultats (que ce soit sous la forme de documents ou de substituts de ceux-ci) et qu'il doit évaluer si ces résultats sont appropriés ou pertinents en fonction de la recherche effectuée ou de son besoin informationnel.

Le jugement de pertinence est généralement considéré sous l'angle de la subjectivité en raison du rôle inhérent du facteur humain. Pour Harter,

"Parce que les juges sont des être humains, leurs propres antécédents, expériences, connaissances, créativité et idées, et comment ces derniers affectent leur état mental courant, ils sont exercés à de telles questions; leurs jugements doivent refléter leurs différences individuelles et subjectives. »

(1992, 612, notre trad.)

Pour Mizzaro (1997), le jugement de pertinence est l'assignation d'une valeur de pertinence par un juge à un moment donné dans le temps. Selon lui, le jugement peut être catégorisé selon cinq dimensions: le type de pertinence jugé, le type de juge, ce qu'évalue le juge (information, document ou substitut), ce que peut utiliser le juge pour exprimer son jugement (problème, requête booléenne, etc.) et le moment où est effectué le jugement. Ces dimensions renvoient à son modèle d'ordre partiel décrit plus haut.

\section{Critères d'évaluation}

Les modèles théoriques de la pertinence que nous avons présentés n'identifient pas les critères utilisés par l'usager pour juger de la pertinence des résultats de recherche. Ce sont pourtant ces critères qui permettent de mieux comprendre comment sont évalués les documents et d'avoir ainsi une meilleure idée du succès des systèmes de recherche d'information (Ruthven, Baillie et Elsweiler 2007). Nous présentons ici trois études en lien avec ces critères : les deux premières s'intéressent à des critères spécifiques du jugement de pertinence tandis que la troisième cherche à comprendre la variabi- lité des critères utilisés durant le processus de :acherche d'information.

Nouveauté, topicalité, fiabilité, compréhensibilité et portée

À partir d'une approche cognitive, Xu et Chen (2006) ont étudié cinq facteurs susceptibles d'influencer le jugement de pertinence, à savoir la nouveauté, la topicalité, la fiabilité, la compréhensibilité et la portée. Ces critères ont été choisis en fonction d'un modèle théorique issu du domaine de la communication, la théorie de Grice, qui s'articule autour du concept de coopération dans la communication verbale et non-verbale, c'est-à-dire lorsque l'émetteur et le récepteur coopèrent pour rendre claires leurs significations. Partant de ce principe, $\mathrm{Xu}$ et Chen élaborent leur cadre théorique en définissant cinq critères : la nouveauté est une propriété prenant en compte le caractère inédit ou différent des attentes de l'utilisateur, caractère qui induit un stimulus comme le note Harter dans sa définition de pertinence psychologique (1992) ; la topicalité réfère au degré de rapprochement entre le sujet du document évalué et le sujet d'intérêt de l'utilisateur; la fiabilité renvoie au degré de vérité, d'exactitude ou de crédibilité de contenu du document ; la compréhensibilité est le degré de facilité de lecture et de compréhension du document, tel que perçu par l'usager; la portée est définie par la mesure selon laquelle le contenu du document semble approprié par l'usager pour son besoin informationnel.

$\mathrm{Xu}$ et Chen (2006) ont tenté de vérifier, à l'aide d'une analyse psychométrique, le lien d'association entre le jugement de pertinence et ces différents facteurs. Leurs résultats tendent à démontrer que la nouveauté et la topicalité sont des facteurs essentiels au jugement de pertinence, que la compréhensibilité et la fiabilité y sont aussi significativement liées alors que la portée n'est pas un critère de jugement de la pertinence.

\section{Connaissance, intérêt et confiance}

Ruthven, Baillie et Elsweiler (2007) ont cherché à connaitre les effets relatifs de la connaissance, de l'intérêt et de la confiance sur le jugement de la pertinence. Ils notent que l'incohérence dans le jugement de pertinence est due à trois raisons principales :

1. La mesure de la pertinence est affectée par la manière même dont elle est effectuée. Par exemple, l'utilisation du texte intégral ou du simple résumé peut grandement influer sur le résultat du jugement ;

2. La pertinence est affectée par le contexte de recherche. Par exemple, le jugement tient généralement compte d'une interdépendance entre les différents documents évalués. Mizzaro (1997) mentionne d'ailleurs l'effet de contexte décrit par Eisenberg (1986), c'est-à-dire la possibilité 
que le jugement de pertinence du document puisse être affecté par d'autres documents évalués :

3. La pertinence est affectée par le contexte du chercheur. La recherche étant un processus intellectuel, elle est grandement influencée par les caractéristiques du chercheur ou de la relation entre la tâche de recherche et les documents récupérés.

Ruthven et ses collègues présentent la piste HARD (High Accuracy Retrieval from Documents) de TREC comme point de départ pour leur étude. Le but de la piste HARD est d'étudier les stratégies possibles d'utilisation d'éléments de contexte de l'utilisateur pour améliorer le repérage d'information. Dans le protocole du concours, un formulaire de clarification permet de récupérer des informations spécifiques sur l'évaluateur des documents. Ruthven, Baillie et Elsweiler, lors de leur participation au concours, ont utilisé ce formulaire pour recueillir des informations sur la familiarité avec le sujet de la recherche, la confiance dans le jugement ainsi que l'intérêt par rapport au sujet de la recherche.

Bien que les chercheurs n'aient pu approfondir leur investigation à l'aide de données qualitatives en raison du protocole de TREC, les résultats ont semblé démontrer que les juges ayant une connaissance significative ou un intérêt marqué pour le sujet (topic) de recherche, ou une grande confiance dans leur jugement, ont tendance à relever davantage de documents comme étant pertinents.

\section{Critères de jugement et évolution} du processus de recherche

Taylor (2012) s'est attardé à la variabilité des critères de jugement à différents stades du processus de recherche. Selon lui, le jugement de pertinence est soumis à une panoplie de critères qui eux-mêmes évoluent de manière dynamique dans le temps, en fonction de changements d'états cognitifs de l'usager.

Dans deux études quantitatives distinctes, Taylor a analysé les critères de jugement identifiés par les répondants en les comparant avec les stades du processus de recherche. Ces stades sont ceux décrits par Wilson (1999) qui combine le modèle comportemental de Ellis (1997) et les stades de recherche de Kuhlthau (1991, 1993). Quant aux critères utilisés dans l'étude, Taylor en dénombre une vingtaine à partir de la littérature. Bien que l'étude repose sur un échantillonnage par convenance limité à des étudiants d'un même domaine (l'administration) et d'un même cours universitaire, ce qui affaiblit la capacité de généralisation des résultats, Taylor (2012) note une manifestation statistiquement forte de la nature dynamique de la pertinence, ce qui suggère l'importance d'avoir des systèmes de repérage de l'information dynamiques et adaptatifs.

\section{Contexte et jugement de pertinence}

Nous venons de le voir avec les études de Taylor (2012), de $\mathrm{Xu}$ et Chen (2006) et de Ruthven, Baillie et Elsweiler (2007), la pertinence et le jugement qu'en fait l'usager sont de nature dynamique et impliquent une multitude de critères d'évaluation tout au long du processus de recherche. Le contexte, qu'il soit défini au niveau du système de recherche lui-même ou selon des caractéristiques propres à l'usager, influence aussi ces critères, comme le font remarquer Ruthven et ses collègues:

"Le contexte, en ce sens, peut signifier toute information qui pourrait influencer la manière dont un évaluateur détermine la pertinence d'un document; le contexte pourrait inclure de l'information sur les évaluateurs eux-mêmes (par exemple la familiarité avec le sujet ou l'expérience d'évaluation), de l'information sur le besoin informationnel sous-jacent au sujet (par exemple pourquoi l'information est requise) ou toute autre question qui pourrait aider à décider quels documents l'évaluateur pourrait évaluer comme pertinents."

( 2007,487 , notre trad.).

Ruthven, Baillie et Elsweiler (2007) mentionnent d'ailleurs, sans équivoque, l'importance que joue le contexte dans le mécanisme de jugement, comme nous l'avons souligné plus haut.

On peut facilement rattacher les types de contexte évoqués par Ruthven et ses collègues aux strates de contexte "structures inter-objets ", "interactions » et "situation / tâche / etc. » de Ingwersen et Järvelin (Figure 2), de même qu'au modèle stratifié d'interactions de Saracevic (Figure 3). Dans ces deux modèles, les interactions entre l'usager et le système sont tributaires de facteurs contextuels, expliquant la complexité $\mathrm{du}$ processus de jugement de pertinence. Or, les interactions constituent ici le point de jonction entre les " contexte du chercheur » et " contexte de recherche".

Comme nous l'avons vu, le contexte du chercheur, tel que mentionné par Ruthven, Baillie et Elsweiler (2007), fait référence aux caractéristiques propres du chercheur, sa connaissance, ses intérêts et la tâche qu'il réalise mais aussi à la situation et à l'environnement dans lequel il évolue.

Quant au contexte de recherche, qui englobe à la fois les composantes technologiques et les collections de documents ou ressources informationnelles, il influence directement les facteurs de topicalité, nouveauté et fiabilité. En effet, le chercheur spécialisé, confronté à une multiplicité de plateformes de recherche, choisira probablement celles dont la collection se rapproche de ses intérêts de recherche immédiats, favorisant la découverte de documents qu'il jugera plus pertinents. De même, la nature dynamique des collections peut accroître l'effet de nouveauté, facteur important pour 
le jugement de pertinence (Xu et Chen 2006). Enfin, la qualité des articles et la renommée des revues et des auteurs y publiant ne peuvent qu'exercer une influence positive sur la fiabilité d'une collection.

Les relations potentielles entre les divers documents d'une collection, ou le contexte "inter-objets", doivent aussi être considérées comme partie intégrante du contexte de recherche. Bien que certaines de ces relations soient explicites à travers une classification ou un regroupement thématique, elles peuvent néanmoins être peu visibles pour l'usager. Or, l'effet de contexte de Eisenberg mentionné par Mizzaro (1997), soit l'influence des relations entre les documents dans le jugement de pertinence, fait ressortir l'importance, pour le chercheur, de pouvoir tirer profit de ces relations afin qu'il puisse maximiser son expérience de recherche.

Enfin, la variabilité du comportement informationnel peut être expliquée par la nature dynamique et multidimensionnelle du contexte. L'étude de Taylor (2012) confirme cette même variabilité dans le jugement de pertinence qui fait partie intégrante du processus de recherche et de repérage de l'information.

\section{Conclusion}

Les concepts de contexte et de pertinence partagent plusieurs caractéristiques et ont été abordés au fil des années selon des épistémologies positivistes, socioconstructivistes et sociotechniques. Le contexte, d'abord décrit comme réalité objective, s'est mué en un ensemble de composantes humaines et sociales auxquelles se sont liées des réalités plus larges comme l'environnement économique, sociétal ou technique, ce qu'Ingwersen et Järvelin nomment l'infrastructure (2005), pour finalement devenir une "écologie de l'information ». Cette écologie implique un caractère dynamique et des interactions entre divers types de contexte (Courtright 2007).

De la même manière, la notion de pertinence a été envisagée de manière algorithmique puis, à la lumière des études sur le comportement informationnel, a été définie selon des "manifestations" prenant davantage en compte des réalités psychologiques, cognitives, sociales ou situationnelles. Depuis le milieu des années 1990, on assiste à une intégration des modèles du comportement informationnel dans les modèles théoriques du concept de pertinence. Ces nouveaux modèles, comme celui de Saracevic (1996) ou de Borlund (2003), considèrent la pertinence en fonction des interactions entre l'usager, le système et l'environnement.

La mesure de la pertinence passe par un processus de jugement effectué par l'usager ou un juge lorsqu'il est question d'évaluer un système. Or, nous l'avons vu, le jugement peut être affecté par différentes dimensions du contexte, soit le contexte du chercheur et le contexte du système de recherche (Ruthven, Baillie et Elsweiler 2007). De plus, les critères de jugement variant selon le stade du processus de recherche (Taylor 2012), il nous apparaît évident que cette variabilité est le résultat des caractéristiques multidimensionnelles et dynamiques de la pertinence et du contexte.

À la lumière de cette analyse, nous considérons que la prise en compte de certaines dimensions du contexte dans les systèmes de repérage de l'information est d'un grand intérêt pour le développement de systèmes de recherche de nature exploratoire, comme le mentionnent d'ailleurs White et Roth (2009). De tels systèmes devraient être en mesure d'offrir un meilleur support à l'usager quant à l'évaluation des résultats de recherche, tirant ainsi profit à la fois d'une certaine connaissance du contexte de l'usager mais aussi du contexte de recherche, et ce, de manière dynamique. (-)

\section{Sources consultées}

Borlund, Pia. 2003. The concept of relevance in IR. Journal of the American Society for Information Science and Technology 54(10) : 913-925.

Buckland, Michael K. 1991. Information as thing. Journal of the American Society for Information Science 42(5) : 351-360.

Cosijn, Erica et Peter Ingwersen. 200o. Dimensions of relevance. Information Processing \& Management 36(4) : 533-550.

Courtright, Christina. 2007. Context in information behavior research. Annual Review of Information Science and Technology 41(1) : 273-306.

Harter, Stephen P. 1992. Psychological relevance and information science. Journal of the American Society for Information Science 43(9) : 602-615.

Ingwersen, Peter. 1996. Cognitive perspectives of information retrieval interaction : elements of a cognitive IR theory. Journal of Documentation 52(1) : 3-50.

Ingwersen, Peter et Kalervo Järvelin. 2005. The Turn : Integration of Information Seeking and Retrieval in Context. The Information Retrieval Series (Vol. 18). Dordrecht : Springer.

Kuhlthau, Carol C. 1991. Inside the search process : Information seeking from the user's perspective. Journal of the American Society for Information Science 42(5) : 361-371.

Marchionini, Gary. 2006. Exploratory search : from finding to understanding. Commun. ACM 49(4) : 41-46.

Mizzaro, Stefano. 1997. Relevance : The whole history. Journal of the American Society for Information Science 48(9) : 810-832.

Mizzaro, Stefano. 1998. How many relevances in information retrieval ? Interacting with Computers $10(3): 303-320$.

Ruthven, Ian, Mark Baillie, et David Elsweiler. 2007. The relative effects of knowledge, interest and confidence in assessing relevance. Journal of Documentation 63(4) : 482-504.

Saracevic, Tefko. 1975. RELEVANCE : A review of and a framework for the thinking on the notion in information science. Journal of the American Society for Information Science 26(6) : 321-343.

Saracevic, Tefko. 1996. Relevance reconsidered. Proceedings of the second conference on conceptions of library and information science (CoLIS 2) (pp. 201-218). <https ://comminfo.rutgers. edu/ tefko/CoLIS2_1996.doc> (consulté le 7 mai 2012).

Taylor, Arthur. 2012. User relevance criteria choices and the information search process. Information Processing \& Management 48(1): 136-153.

White, Ryan W. et Resa A. Roth. 2009. Exploratory Search : Beyond the Query-Response Paradigm. Synthesis Lectures on Information Concepts, Retrieval, and Services 1(1) : 1-98.

Wilson, Tom D. 1999. Models in Information Behaviour Research. Journal of Documentation 55(3) : 249-270.

$\mathrm{Xu}$, Yunjie (Calvin) et Zhiwei Chen. 2006. Relevance judgment : What do information users consider beyond topicality? Journal of the American Society for Information Science and Technology 57(7) : 961-973. 